\title{
Problems with and a system to eliminate single-primer PCR product contamination in simple sequence repeat molecular marker-assisted selection in soybean
}

\author{
J. Ma, S.C. Guan, D. Yao, Y.F. Wei and P.W. Wang \\ Biotechnology Center, Jilin Agricultural University, Changchun, P.R. China \\ Corresponding author: P.W. Wang \\ E-mail: winter0106@163.com
}

Genet. Mol. Res. 10 (3): 1659-1668 (2011)

Received March 11, 2011

Accepted July 12, 2011

Published August 5, 2011

DOI http://dx.doi.org/10.4238/vol10-3gmr1366

\begin{abstract}
Polymerase chain reaction (PCR) provides a foundation for simple sequence repeat molecular marker-assisted selection (SSR MAS) in soybean. This PCR system and its various conditions have been optimized by many researchers. However, current research on the optimization of the PCR system focuses on double-primer PCR products. We compared single- and double-SSR primer PCR products from 50 soybean samples and found that the use of single-PCR primers in the reaction system can lead to amplified fragments of portions of the SSR primers in the PCR process, resulting in both false-positives and fragment impurity of double-primer PCR amplification, inconvenient for subsequent analysis. We used "single-primer PCR correction" to eliminate interference caused by single-primer nonspecific PCR amplification and improve PCR quality. Using this method, the precision and success rates of SSR MAS in soybean can be increased.
\end{abstract}

Key words: Soybean; SSR molecular marker-assisted selection; False-positive exclusion; Single-primer PCR correction 


\section{INTRODUCTION}

Polymerase chain reaction (PCR) is the basis for simple sequence repeat molecular marker-assisted selection (SSR MAS) in soybean (Du et al., 2009a; Liang et al., 2010; Liu et al., 2010), and it is critical for experiments to acquire correct PCR products. Many researchers have previously performed various optimizations for the PCR system and conditions, such as annealing temperature, primer specificity and template concentration, to obtain PCR products as correct as possible. However, current research on the optimization of the PCR system focuses on double-primer-based PCR products.

It is known that plant genomic sequences are huge and PCR primers are paired together for use in a single PCR. It is therefore likely that only one primer binds to the genomic DNA or cDNA template and participates in the amplification process. Attention should be paid to the resultant fragment impurity or nonspecific amplification, which is subsequently troublesome in the analysis.

This study compared single- and double-SSR primer PCR products between 50 pairs of soybean and found that single-PCR primers involved in the reaction system can lead to amplified fragments for a portion of the SSR primers in the PCR process, resulting in both nonspecific amplification (false-positives) and fragment impurity of double-primer PCR amplification, causing inconvenience in the subsequent analysis. In this study, "single-primer PCR correction" was preformed to eliminate interference caused by single-primer nonspecific PCR amplification and improve PCR quality. Based on these results, the precision and success rates of SSR MAS in soybean may be increased.

\section{MATERIAL AND METHODS}

\section{Material}

Soybean varieties JiNong27 and TongNong13 and their F1 were provided by the Biotechnology Center of Jilin Agricultural University. PrimeSTAR ${ }^{\mathrm{TM}}$ HS DNA Polymerase, dNTP mix, DNA Gel Extraction kit, DNA marker, and Genomic DNA Extraction kit were purchased from TaKaRa (Dalian, China). Other biochemical reagents, including acrylamide, methylene acrylamide, boric acid, ethylenediaminetetraacetic acid (EDTA), sodium hydroxide $(\mathrm{NaOH})$, silver nitrate $\left(\mathrm{AgNO}_{3}\right)$, Tris (hydroxymethyl) aminomethane (Tris), urea, Tris base, ethidium bromide, agarose, yeast extract powder, and tryptone were supplied by AMRESCO (USA).

\section{SSR molecular marker primers}

SSR primer pairs were randomly selected for 40 individual SSRs (Satt66, Satt67, Satt83, Satt100, Satt134, Satt138, Satt142, Satt171, Satt186, Satt199, Satt200, Satt225, Satt226, Satt228, Satt277, Satt291, Satt292, Satt307, Satt316, Satt322, Satt354, Satt356, Satt369, Satt371, Satt416, Satt424, Satt432, Satt443, Satt457, Satt458, Satt520, Satt524, Satt578, Satt593, Satt599, Satt636, Satt656, Satt690, Satt705, and Satt712) from SSR primer sequences on the SoyBase and the Soybean Breeder's Toolbox website (http://soybase.org/ resources/ssr.php). 


\section{Single-/double-primer PCR}

Total DNA was extracted from leaves of JiNong27 nad TongNong13 and their F1 using the Universal Genomic DNA Extraction kit, version 3.0, according to manufacturer instructions $(\mathrm{TaKaRa})$. Nucleic acids were quantitatively measured on a nucleic acid/protein detector (ND-1000, Nano Drop Techonlogies, USA). The PCR system and conditions used in this study referred to the SoyBase and the Soybean Breeder's Toolbox website (http://soybase. org/resources/ssr.php).

The reaction mixture for double-primer amplification of Satt66 (50 $\mu \mathrm{L}$ total) contained: $10.0 \mu \mathrm{L}$ 5X PrimeSTAR buffer, $4.0 \mu \mathrm{L}$ dNTP mix (2.5 mM each dNTP), $1.0 \mu \mathrm{L}$ of each Satt100 P1 and P2 primers $(20 \mu \mathrm{M}$ each), $1.0 \mu \mathrm{L}$ genomic DNA template $(<1 \mu \mathrm{g}), 0.4$ $\mu \mathrm{L}$ PrimeSTAR ${ }^{\mathrm{TM}} \mathrm{HS}$ DNA Polymerase $(2.5 \mathrm{U} / \mu \mathrm{L})$ and $32.6 \mu \mathrm{L} \mathrm{ddH_{2 }} \mathrm{O}$. Cycling conditions comprised 45 cycles of denaturation at $92^{\circ} \mathrm{C}$ for $10 \mathrm{~s}$, annealing at $46^{\circ} \mathrm{C}$ for $10 \mathrm{~s}$ and extension at $68^{\circ} \mathrm{C}$ for $30 \mathrm{~s}$.

The reaction mixture for single-primer amplification of Satt66 P1 (50 $\mu \mathrm{L}$ total) contained: $10.0 \mu \mathrm{L} 5 \mathrm{X}$ PrimeSTAR buffer, $4.0 \mu \mathrm{L}$ dNTP mix (2.5 mM each dNTP), $1.0 \mu \mathrm{L}$ Satt66 P1 primer $(20 \mu \mathrm{M}), 1.0 \mu \mathrm{L}$ genomic DNA template $(<1 \mu \mathrm{g}), 0.4 \mu \mathrm{L}$ PrimeSTAR $^{\mathrm{TM}}$ HS DNA Polymerase $(2.5 \mathrm{U} / \mu \mathrm{L})$ and $33.6 \mu \mathrm{LddH}_{2} \mathrm{O}$. Cycling conditions were the same as for doubleprimer PCR of Satt66.

The reaction mixture and cycling conditions for Satt66 P2 single-primer amplification were the same as for Satt66 P1 single-primer PCR.

The reaction mixture and cycling conditions for single- and double-primer amplification of the other 39 pairs of primers were the same as for Satt66 PCR, except that the annealing temperature was adjusted for individual primers.

\section{Sequencing of PCR products}

The single-/double-primer PCR products of the same length were extracted and ligated into the pMD18T vector, followed by transformation. Sequencing was performed by Beijing SunBiotech Co., Ltd. (Beijing, China).

\section{RESULTS}

In the soybean varieties JiNong27 and TongNong13 and their F1, the Satt67, Satt134, Satt142, Satt228, Satt291, Satt424, Satt443, Satt520 single and double primers produced some fragments of identical length, as shown in Figures 1-8.

As shown in Figure 1, in the male parent Jinong27, Satt67 double-primer amplified fragment A, and the single-primer P2 amplified fragment B. Fragments A and B had the same length. In the female parent Tongnong13, Satt67 double-primer fragment $\mathrm{H}$ and the singleprimer P2 fragment $G$ had the same length. F1 Satt67 double-primer fragment C and the single-primer P2 fragment $\mathrm{D}$ had the same length; double-primer fragment $\mathrm{E}$ and the singleprimer $\mathrm{P} 2$ fragment $\mathrm{F}$ had the same length. There were two possibilities: 1) $\mathrm{A}$ and $\mathrm{B}, \mathrm{C}$ and $\mathrm{D}$, $\mathrm{E}$ and $\mathrm{F}, \mathrm{H}$, and $\mathrm{G}$ were the same fragments, respectively, and they were single-primer P2 PCR products; 2) A, C, E, and $\mathrm{H}$ were impurities, namely B contaminated A, D contaminated C, F contaminated $\mathrm{E}$, and $\mathrm{G}$ contaminated $\mathrm{H}$. 


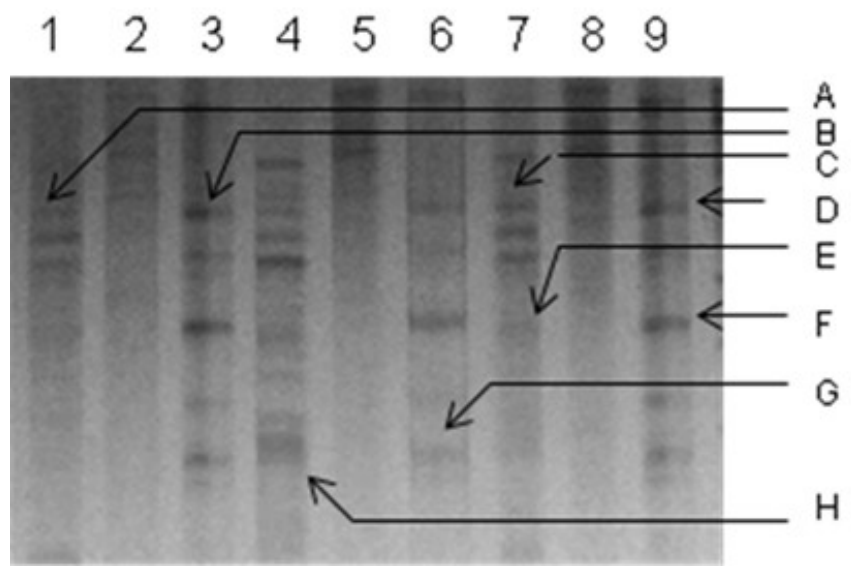

Figure 1. PCR amplification fragments using Satt67 single and double primers for JiNong27 (males) and TongNong13 (females) and hybrid generation. Lane $1=$ using Satt67 double primer (P1 and P2) for JiNong27; lane 2 = using P1 single primer for JiNong27; lane 3 = using P2 single primer for JiNong27; lane 4 = using Satt67 double primer (P1 and P2) for TongNong13; lane $5=$ using P1 single primer for TongNong13; lane $6=$ using P2 single primer for TongNong13; lane $7=$ using Satt67 double primer (P1 and P2) for F1; lane $8=$ using P1 single primer for F1; lane 9 = using P2 single primer for F1. For A-H explanations, see Results.

For the Satt134 single- and double-primer PCR products, the male parent double-primer fragment A had the same length as the single-primer P1 fragment B; the double-primer fragment C and single-primer P1 fragment D had the same length. The female parent double-primer fragment $\mathrm{E}$ and the single-primer P1 fragment F had the same length, as shown in Figure 2.

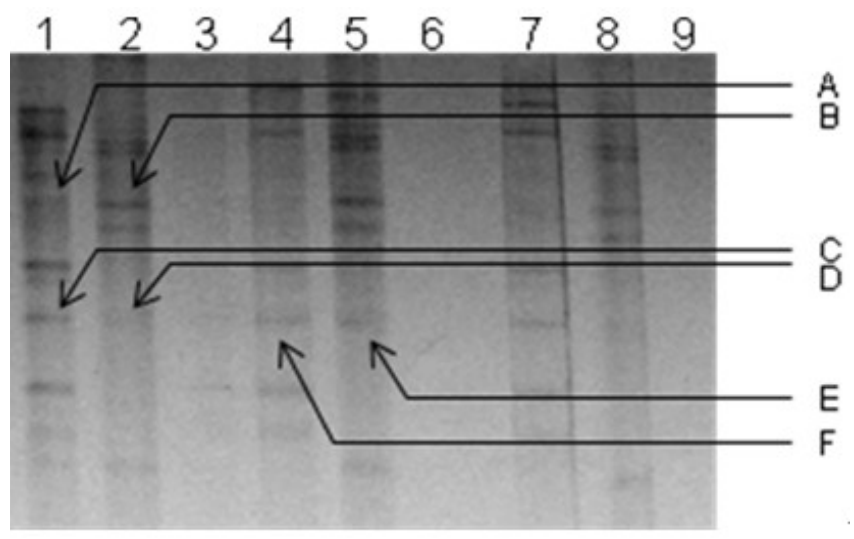

Figure 2. PCR amplification fragments using Satt134 single and double primers for JiNong27 (males) and TongNong13 (females) and hybrid generation. Lane 1 = using Satt134 double primer for JiNong 27; lane $2=$ using P1 primer for JiNong 27; lane 3 = using P2 primer for JiNong 27; lane 4 = using Satt134 double primer for TongNong13; lane $5=$ using P1 primer for TongNong13; lane $6=$ using P2 primer for TongNong13; lane $7=$ using Satt 134 double primer for $\mathrm{F} 1$; lane $8=$ using P1 primer for F1; lane $9=$ using P2 primer for F1. For A-F explanations, see Results. 
For the Satt142 single- and double-primer PCR products, the male parent doubleprimer fragment A had the same length as the single-primer P1 fragment B; the doubleprimer fragment $\mathrm{H}$ and the single-primer P2 fragment $\mathrm{G}$ had the same length. The female parent double-primer fragment $\mathrm{F}$ and the single-primer $\mathrm{P} 1$ fragment $\mathrm{E}$ had the same length. The F1 double-primer fragment D and the single-primer P1 fragment $\mathrm{C}$ had the same length, as shown in Figure 3.

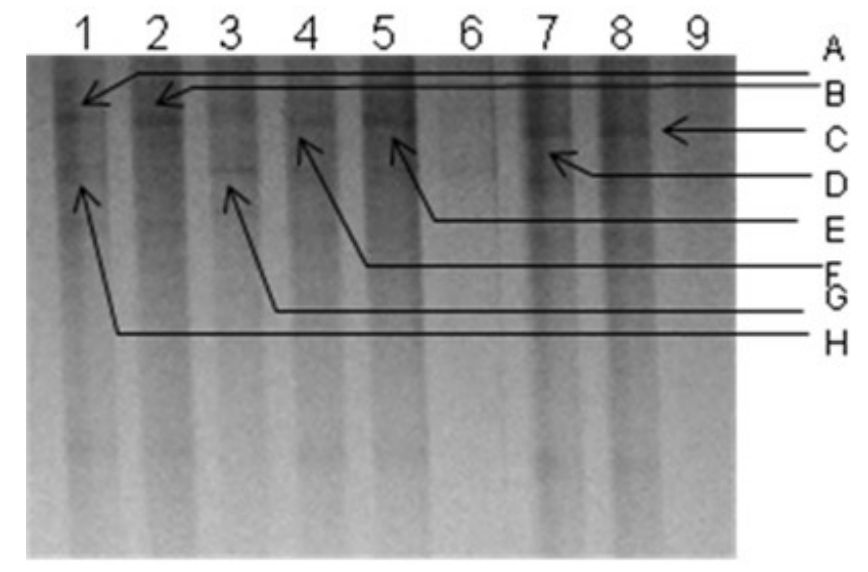

Figure 3. PCR amplification fragments using Satt142 single and double primers for JiNong27 (males) and TongNong13 (females) and hybrid generation. Lane $1=$ using Satt142 double primer for JiNong 27; lane 2 = using P1 primer for JiNong 27; lane 3 = using P2 primer for JiNong 27; lane 4 = using Satt142 double primer for TongNong13; lane $5=$ using P1 primer for TongNong13; lane $6=$ using P2 primer for TongNong13; lane 7 = using Satt225 double primer for F1; lane $8=$ using P1 primer for F1; lane $9=$ using P2 primer for F1. For A-H explanations, see Results.

For the Satt228 single- and double-primer PCR products, the male parent doubleprimer fragment A had the same length as the single-primer P2 fragment B; the doubleprimer fragment $\mathrm{J}$ and the single-primer P1 fragment I had the same length. The female parent double-primer fragment $\mathrm{D}$ had the same length as the single-primer P2 fragment $\mathrm{C}$; the double-primer fragment $\mathrm{H}$ and single-primer $\mathrm{P} 1$ fragment $\mathrm{G}$ had the same length. The F1 double-primer fragment $\mathrm{D}$ and single-primer $\mathrm{P} 1$ fragment $\mathrm{C}$ had the same length, as shown in Figure 4.

For the Satt291 single- and double-primer PCR products, the male parent doubleprimer fragment A and single-primer P1 fragment B had the same length. The F1 doubleprimer fragment D and the single-primer $\mathrm{P} 1$ fragment $\mathrm{C}$ had the same length, as shown in Figure 5.

For the Satt424 single- and double-primer PCR products, the male parent doubleprimer fragment A, single-primer P1 fragment B and single-primer P2 fragment C had the same length; the double-primer fragment D and single-primer P1 fragment $\mathrm{E}$ had the same length; the double-primer fragment $\mathrm{H}$ and single-primer P1 fragment I had the same length. The female parent double-primer fragment $\mathrm{F}$ had the same length as the single-primer P1 fragment $\mathrm{G}$; the double-primer fragment $\mathrm{J}$ and the single-primer $\mathrm{P} 1$ fragment $\mathrm{M}$ had the same 


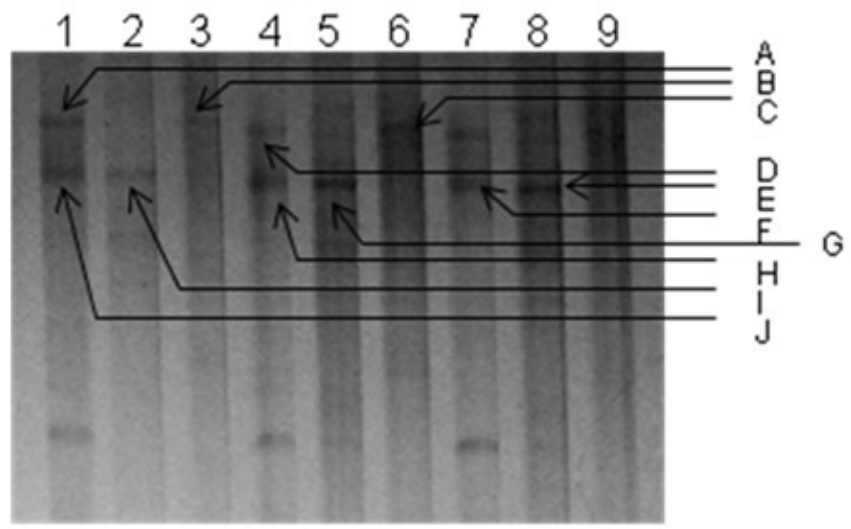

Figure 4. PCR amplification fragments using Satt228 single and double primers for JiNong27 (males) and TongNong 13 (females) and hybrid generation. Lane $1=$ using Satt228 double primer for JiNong27; lane 2 = using P1 primer for JiNong27; lane 3 = using P2 primer for JiNong27; lane $4=$ using Satt228 double primer for TongNong13; lane $5=$ using P1 primer for TongNong13; lane $6=$ using P2 primer for TongNong13; lane $7=$ using Satt228 double primer for F1; lane 8 = using P1 primer for F1; lane 9 = using P2 primer for F1. For A-J explanations, see Results.

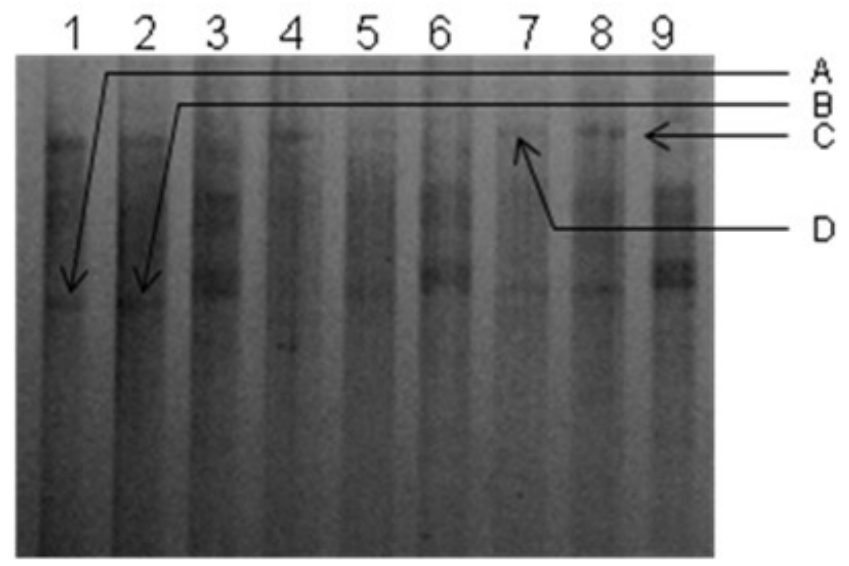

Figure 5. PCR amplification fragments using Satt291 single and double primers for JiNong27 (males) and TongNong 13 (females) and hybrid generation. Lane $1=$ using Satt291 double primer for JiNong27; lane 2 = using P1 primer for JiNong27; lane 3 = using P2 primer for JiNong27; lane 4 = using Satt291 double primer for TongNong13; lane $5=$ using P1 primer for TongNong13; lane $6=$ using P2 primer for TongNong13; lane 7 = using Satt291 double primer for F1; lane $8=$ using P1 primer for F1; lane $9=$ using P2 primer for F1. For A-D explanations, see Results.

length. The F1 double-primer fragment $\mathrm{L}$ and the single-primer $\mathrm{P} 1$ fragment $\mathrm{K}$ had the same length, as shown in Figure 6.

For the Satt443 single- and double-primer PCR products, the male parent doubleprimer fragment A and single-primer P2 fragment B had the same length; the double-primer fragment $\mathrm{C}$ and the single-primer $\mathrm{P} 1$ fragment $\mathrm{D}$ had the same length. The female parent double-primer fragment $\mathrm{E}$ and single-primer $\mathrm{P} 1$ fragment $\mathrm{F}$ had the same length, as shown in Figure 7. 


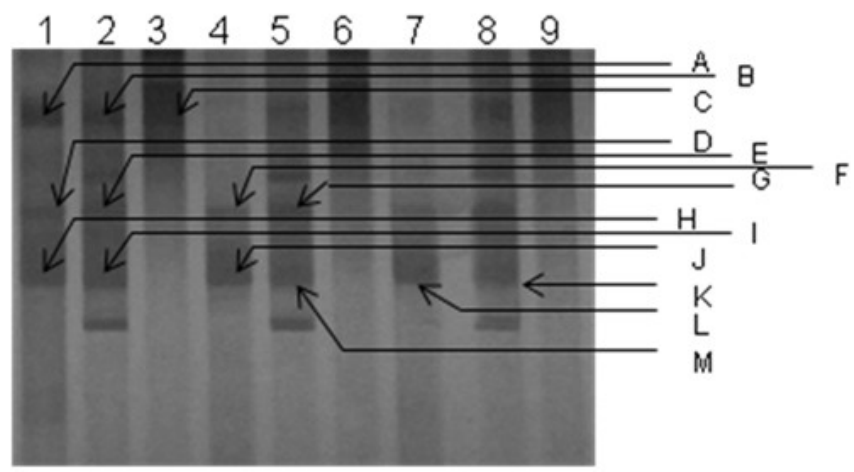

Figure 6. PCR amplification fragments using Satt 424 single and double primers for JiNong27 (males) and TongNong 13 (females) and hybrid generation. Lane $1=$ using Satt424 double primer for JiNong27; lane 2 = using P1 primer for JiNong27; lane 3 = using P2 primer for JiNong27; lane $4=$ using Satt424 double primer for TongNong13; lane $5=$ using P1 primer for TongNong13; lane $6=$ using P2 primer for TongNong13; lane $7=$ using Satt424 double primer for F1; lane 8 = using P1 primer for F1; lane 9 = using P2 primer for F1. For A-M explanations, see Results.

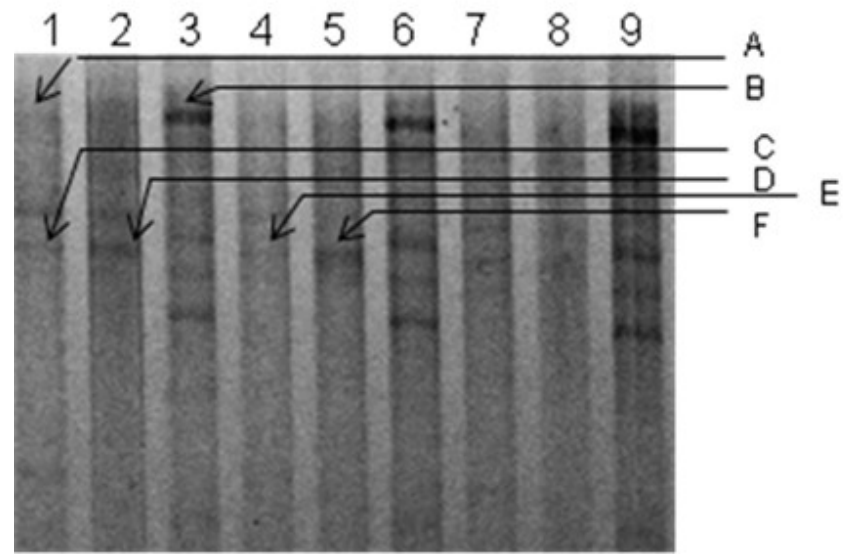

Figure 7. PCR amplification fragments using Satt443 single and double primers for JiNong 27 (males) and TongNong13 (females) and hybrid generation. Lane 1 = using Satt443 double primer for JiNong27; lane 2 = using P1 primer for JiNong27; lane 3 = using P2 primer for JiNong27; lane 4 = using Satt443 double primer for TongNong13; lane 5 = using $\mathrm{P} 1$ primer for TongNong13; lane $6=$ using $\mathrm{P} 2$ primer for TongNong13; lane $7=$ using Satt443 double primer for F1; lane $8=$ using P1 primer for F1; lane $9=$ using P2 primer for F1. For A-F explanations, see Results.

For the Satt520 single- and double-primer PCR products, the male parent double-primer fragment $\mathrm{A}$ and single-primer $\mathrm{P} 1$ fragment $\mathrm{B}$ had the same length. The female parent double-primer fragment $\mathrm{C}$ and single-primer $\mathrm{P} 1$ fragment $\mathrm{E}$ had the same length, as shown in Figure 8.

Upon the analysis of sequencing outcomes, the Satt424 single- and double-primer PCR products of the same length were extracted and ligated into the pMD18T vector, followed by transformation to Escherichia coli and sequencing. The sequencing outcomes showed that the male parent double-primer fragment A was a mixture consisting of the single-primer P1 
fragment B and the single-primer P2 fragment $\mathrm{C}$, instead of double-primer (P1 and P2) PCR products; the single-primer $\mathrm{P} 1$ fragment $\mathrm{E}$ and I contaminated the double-primer fragment $\mathrm{D}$ and $\mathrm{H}$, respectively. In the female parent, the single-primer P1 fragments $\mathrm{G}$ and $\mathrm{M}$ contaminated the double-primer fragment $\mathrm{F}$ and J, respectively. In F1, the single-primer P1 fragment $\mathrm{K}$ contaminated the double-primer fragment $\mathrm{L}$. These results demonstrated that in the SSR marker double-primer PCR products in soybean, some fragments do not yield pure doubleprimer PCR products, they were contaminated by the single-primer fragment impurities, and they were actually the single-primer PCR products.

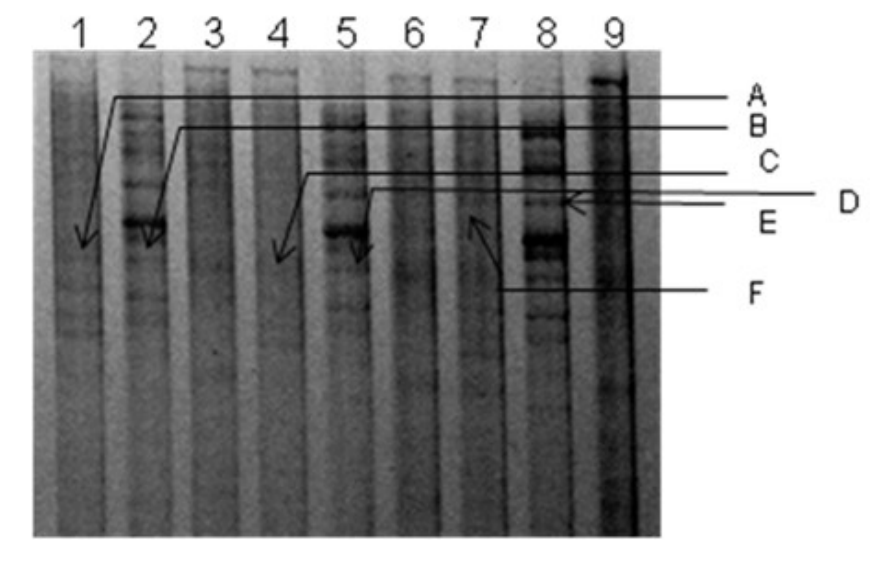

Figure 8. PCR amplification fragments using Satt520 single and double primers for JiNong27 (males) and TongNong13 (females) and hybrid generation. Lane $1=$ using Satt520 double primer for JiNong27; lane $2=$ using P1 primer for JiNong27; lane 3 = using P2 primer for JiNong27; lane $4=$ using Satt520 double primer for TongNong13; lane $5=$ using P1 primer for TongNong13; lane $6=$ using P2 primer for TongNong13; lane 7 = using Satt520 double primer for F1; lane $8=$ using P1 primer for F1; lane $9=$ using P2 primer for F1. For A-F explanations, see Results.

\section{DISCUSSION}

PCR technology is a potent tool in molecular biology (Ferreira et al., 2004; Ning et al., 2007; Amar et al., 2011) and also plays an important role in SSR MAS in soybean (Wen et al., 2008; Du et al., 2009b; Sayama et al., 2009). The single primers were set up in the PCR process to determine nonspecific amplification, false-positives and the occurrence of mixed single-/double-primer fragments in double-primer PCR; this procedure was called the "singleprimer PCR correction" (Ma et al., 2011). We found that in SSR MAS in soybean, even the use of strict PCR conditions can still result in nonspecific amplification, false-positives and mixed single-/double-primer amplification fragments caused by single-primer PCR amplification. The nonspecific amplification and single-primer fragment contamination were difficult to overcome by optimizing PCR conditions (Ma et al., 2011).

In fact, nonspecific amplification and impure fragments are inevitable because the genome is huge and because some single primers in the PCR process can bind to genomic DNA or cDNA templates and result in fragments that are indistinguishable from those obtained in double-primer PCR, on an agarose or polyacrylamide gel under the same PCR 
conditions. Thus, it is detrimental to SSR MAS breeding. In addition, scientists have differences of opinion regarding parent selection for breeding and they may face unknown sequences as the known SSR primers are used. Due to the lack of a known sequence reference, nonspecific amplification and the mixed single-/double-primer amplification fragments caused by the single-primer PCR amplification should be of concern, also when scientists develop new SSR primers.

During SSR MAS, sequences obtained from target gene cloning are verified by gene sequencing. However, when recycling products mix with PCR products of single-primer amplification, direct gene sequencing of PCR products cannot succeed. Randomness occurs in the selection of a single-bacterial colony for gene sequencing after the fragments of interest are ligated into the pMD18-T vector and transformed. This factor could have serious effects on subsequent experiments.

In view of the above analysis, we recommend the setup of "single-primer PCR correction" both in SSR MAS in soybean and in the development of new SSR primers to verify whether the fragments obtained are nonspecific amplification or if they are contaminated with impure fragments. Once the single-/double-primer PCR fragments are seen in the same position of the gel, direct gene sequencing of the double-primer PCR products is not recommended. It should compare the grayscale of single-/double-primer PCR fragments and estimate the molar ratio. After ligation into the pMD18-T vector and gene transfer, the molar counts of products with a larger grayscale were divided by the molar counts of products with a smaller grayscale, and single bacterial colonies were selected in the number at least over the mole ratio. The universal primer for the pMD18-T vector (such as M13, T7, and SP6) co-operating with the specific primers we designed was used to screen and remove false-positives (for example, if an upstream primer produces mismatches, downstream and universal primers can be used) and then sequencing was performed. All additional procedures are intended to eliminate falsepositives as best as possible and improve the precision of the experiments.

\section{ACKNOWLEDGMENTS}

Research supported by the National Natural Science Foundation of China (\#30971805) and the Young Scientists Foundation of JILIN Province in China (\#20100145).

\section{REFERENCES}

Amar MH, Biswas MK, Zhang Z and Guo WW (2011). Exploitation of SSR, SRAP and CAPS-SNP markers for genetic diversity of Citrus germplasm collection. Sci. Hortic. 128: 220-227.

Du W, Yu DY and Fu SX (2009a). Analysis of QTLs for the trichome density on the upper and downer surface of leaf blade in soybean [Glycine max (L.) Merr.]. Agric. Sci. China 8: 529-537.

Du W, Wang M, Fu S and Yu D (2009b). Mapping QTLs for seed yield and drought susceptibility index in soybean (Glycine $\max$ L.) across different environments. J. Genet. Genomics 36: 721-731.

Ferreira AM, Vitor RW, Carneiro AC, Brandao GP, et al. (2004). Genetic variability of Brazilian Toxoplasma gondii strains detected by random amplified polymorphic DNA-polymerase chain reaction (RAPD-PCR) and simple sequence repeat anchored-PCR (SSR-PCR). Infect. Genet. Evol. 4: 131-142.

Liang HZ, Yu YL, Wang SF, Lian Y, et al. (2010). QTL mapping of isoflavone, oil and protein contents in soybean (Glycine max L. Merr.). Agric. Sci. China 9: 1108-1116.

Liu YL, Li YH, Zhou GA, Uzokwe N, et al. (2010). Development of soybean EST-SSR markers and their use to assess genetic diversity in the subgenus soja. Agric. Sci. China 9: 1423-1429.

Ma J, Wang PW, Yao D, Wang YP, et al. (2011). Single-primer PCR correction: a strategy for false-positive exclusion. Genet. Mol. Res. 10: 150-159. 
Ning SP, Xu LB, Lu Y, Huang BZ, et al. (2007). Genome composition and genetic diversity of Musa germplasm from China revealed by PCR-RFLP and SSR markers. Sci. Hortic. 114: 281-288.

Sayama T, Nakazaki T, Ishikawa G, Yagasaki K, et al. (2009). QTL analysis of seed-flooding tolerance in soybean (Glycine max [L.] Merr.). Plant Sci. 176: 514-521.

Wen ZX, Zhao TJ, Zheng YZ, Liu SH, et al. (2008). Association analysis of agronomic and quality traits with SSR markers in Glycine max and Glycine soja in China: I. population structure and associated markers. Acta Agron. Sin. 34: 1169-1178. 